\title{
A New Method of Intracranial Pressure Monitoring by EEG Power Spectrum Analysis
}

\author{
Hui Chen, Jian Wang, Sizhong Mao, Weiwei Dong, Hao Yang
}

\begin{abstract}
Objectives: To investigate the feasibility of Electroencephalogram (EEG) power spectrum analysis as a noninvasive method for monitoring intracranial pressure (ICP). Methods: The EEG signals were recorded in 62 patients (70 cases) with central nervous system (CNS) disorders in our hospital. By using self-designed software, EEG power spectrum analysis was conducted and pressure index (PI) was calculated automatically. Intracranial pressure was measured by lumbar puncture (LP). Results: We found a significant negative correlation between PI and ICP $(r=-0.849, p<0.01)$. Conclusions: The PI obtained from EEG analysis is correlated with ICP. Analysis of specific parameters from EEG power spectrum might reflect the ICP.
\end{abstract}

RÉSUMÉ: Une nouvelle méthode de surveillance de la pression intracrânienne par analyse des spectres de puissance de l'EEG. Objectifs : Le but de l'étude était d'évaluer la faisabilité de l'analyse des spectres de puissance de l'EEG comme méthode non effractive de surveillance de la pression intracrânienne (PIC). Méthode : Les signaux EEG ont été enregistrés chez 62 patients (70 observations) atteints de troubles du système nerveux central (SNC) dans notre hôpital. Nous avons effectué une analyse des spectres de puissance de l'EEG et l'indice de pression (IP) a été calculé automatiquement. La pression intracrânienne a été mesurée par ponction lombaire. Résultats : Nous avons observé une corrélation négative significative entre l'IP et la PIC $(r=-0,849 ; p<0,01)$. Conclusions : L'IP obtenu de l'analyse de l'EEG était corrélé à la PIC. L'analyse de paramètres spécifiques des spectres de puissance de l'EEG pourrait refléter la PIC.

Can J Neurol Sci. 2012; 39: 483-487

At present, intracranial pressure (ICP) can be reliably measured directly by invasive techniques such as ventriculostomy tube ${ }^{1,2}$, which require special equipment for the placement of monitoring probes. The most common side effect is intracranial infection. ${ }^{3-5}$ Lumbar puncture (LP) is one of the most common methods of ICP measurement, however, when ICP is very high, it should be performed with caution to avoid inducing brain herniation which may lead to death. ${ }^{6}$ In recent years, some noninvasive ICP monitoring methods have been reported, including transcranial doppler, ${ }^{7-9}$ jugular venous oxygen concentration monitoring, measurement of tympanic membrane displacement, ${ }^{10,11}$ and retinal venous pressure measurement, however, none of these methods is sufficiently accurate to be allowed for routine clinical use.

As we know, it is now possible to record and monitor the continuous digital EEG, and continuous EEG monitoring provides dynamic information about brain function. ${ }^{12}$ Electroencephalogram (EEG) power spectrum is a method of EEG signal analysis which provides more accurate information for determining brain functions than traditional EEG techniques. ${ }^{13,14}$ Electroencephalogram power spectrum has been used to assess the level of consciousness and the depth of anesthesia. ${ }^{15}$ Gradual increase of ICP will lead to change of cerebral perfusion pressure. Decreased cerebral perfusion pressure will reduce the cerebral blood flow $(\mathrm{CBF})$, which can cause metabolic injury and ischemic damage of brain tissues, hence the patient will show different degrees of impaired level of consciousness. ${ }^{16}$ Since EEG power spectrum analysis can be used to evaluate the consciousness level, it may be used to reflect the changes in ICP?

In a previous animal experiment (unpublished work), we made a porcine model of cerebral hemorrhage by autologous blood. Intracranial pressure was continuously monitored through ventricular puncture. Meanwhile, EEG was recorded and EEG power spectrum was analyzed. The result showed that the

From the Department of Neurology (HC, JW, SM), the Second Affiliated Hospital, Department of Neurology (WD), the First Affiliated Hospital, Chongqing Medical University; College of Electrical Engineering (HY), Chongqing University, Chongqing China.

Received October 27, 2011. Final Revisions Submitted March 2, 2012. Correspondence to: Jian Wang, Department of Neurology, the Second Affiliated Hospital, Chongqing Medical University, Chongqing, 400010, China. Email: jian.wx@163.com 
median frequency and delta ratio were closely correlated with ICP. Therefore we speculated that EEG power spectrum analysis could indirectly reflect ICP change. In the present study, we carried out EEG power spectrum analysis on 70 cases with central nervous system (CNS) diseases from September 2008 to May 2009. The pressure index (PI) was calculated through a selfdesigned software system. Afterwards the correlation between PI and ICP obtained by LP was analyzed.

\section{Patients and Methods}

\section{Patient data}

Sixty-two consecutive patients who were admitted to the neurology hospital ward in our hospital from September 2008 to May 2009 were recruited in the study (Table 1). The study was approved by the Ethics Committee of the School of Medicine, Chongqing Medical University. All patients and guardians of those younger than 18 years were asked to sign a written consent.

Table 1: Clinical diagnosis of patients

\begin{tabular}{lll}
\hline Diagnosis & $\mathrm{N}$ & $\%$ \\
tuberculous meningitis & 12 & 19.4 \\
viral encephalitis & 8 & 12.9 \\
cerebral contusion and laceration & 5 & 8.1 \\
purulent meningitis & 5 & 8.1 \\
intracerebral hemorrhage & 5 & 8.1 \\
metabolic encephalopathy & 4 & 6.5 \\
subarachnoid hemorrhage & 4 & 6.5 \\
cerebral infarction & 4 & 6.5 \\
benign intracranial hypertension & 1 & 1.6 \\
epilepsy & 3 & 4.8 \\
brain tumor & 3 & 4.8 \\
cranial neuritis & 3 & 4.8 \\
facial paralysis herpes & 2 & 3.2 \\
brain stem encephalitis & 1 & 1.6 \\
vasculitis & 1 & 1.6 \\
cerebral venous sinus thrombosis & 1 & 1.6 \\
Total & 62 & \\
\hline & & 100 \\
\hline
\end{tabular}

\section{EEG signals acquisition}

Collection of EEG signals was performed in the supine position right before LP. The EEG data was recorded from electrode positions T3, T4, Fp1 and Fp2; A1, A2 (references) according to the 10-20 electrode international system with the A1000 EEG monitor. The notch filter $(30 \mathrm{~Hz})$ was adopted. The silver chloride electrode was used. The skin was cleaned with an abrasive cleaning fluid and low impedance electrode paste was used to reduce the electrode skin impedance to $<5000 \Omega$. The $3-$ minute original EEG signals (sampling frequency $1000 \mathrm{~Hz}$ ) of each patient were continuously recorded and stored in computer for analysis.

\section{EEG signal analysis and PI calculation}

Electroencephalogram signals with smooth baseline and without any interference and artifact could be used for analysis. We used some signal processing methods including digital filtering and wavelet transform to exclude artifact and interference noise. A total of 20 segments of EEG were randomly chosen for analysis. Each segment contained EEG signals for five seconds. Several parameters could be obtained from power spectrum analysis, including total power, median frequency and delta ratio. Median frequency means the power of the frequency accounting for $50 \%$ of the total power. The frequency limits 0 $4 \mathrm{~Hz}$ are used to define delta power and delta ratio is the ratio of delta power to the sum of alpha power and beta power.

In the original animal experiment we examined which parameters were useful to reflect the decreased cerebral metabolism along with increased ICP. The result showed that compared with other parameters, median frequency and delta ratio were most sensitive in reflecting the changes of ICP. Moreover, we found that 1 / (median frequency $\times$ delta ratio) and ICP had the strongest correlation, so we defined it as "PI" and calculated it automatically using a self-designed software system.

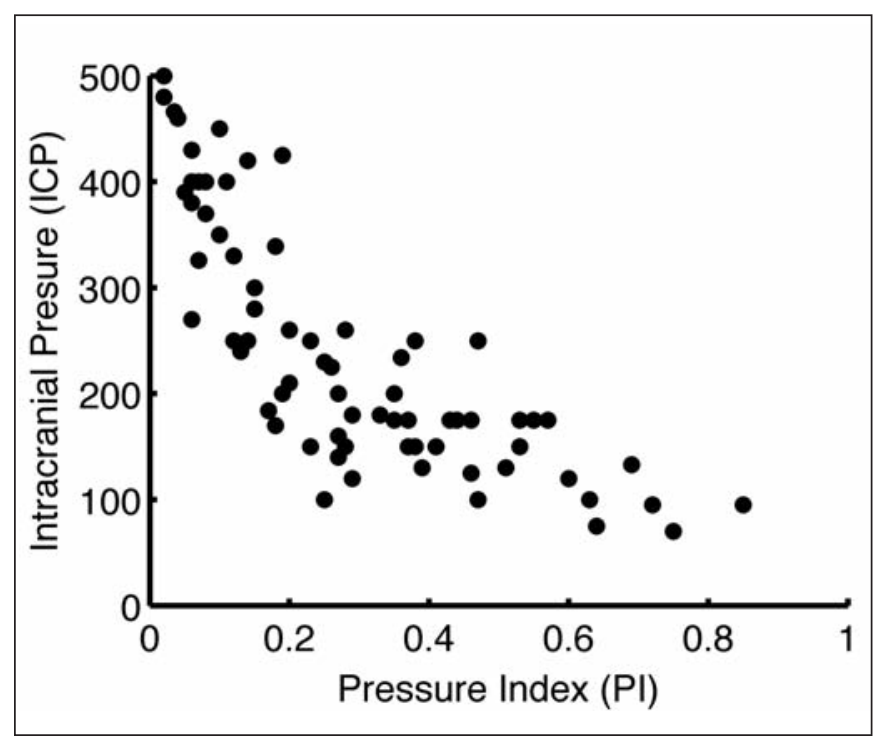

Figure 1: Correlation between PI and ICP in all patients $(p<0.01 ; r=$ $-0.849 ; n=70)$ 


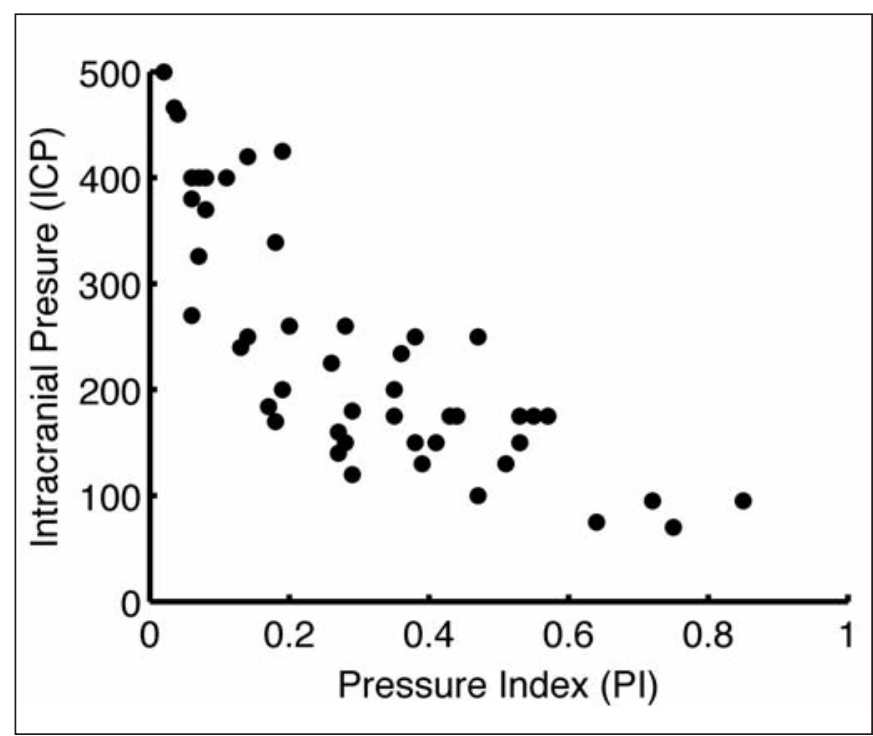

Figure 2: Correlation between PI and ICP in patients with diffuse lesions of CNS $(p<0.01 ; r=-0.815 ; n=47)$

\section{ICP measurement}

Intracranial pressure was measured by LP with the patient in lateral decubitus position immediately after EEG signals acquisition. Lumbar puncture was performed by a neurologist with the same method used in all patients. Increased ICP was defined as an ICP greater than $200 \mathrm{mmH}_{2} \mathrm{O}$.

\section{Statistical analysis}

The data were evaluated by Spearman rank correlation analysis using the Statistical Package for the Social Sciences (SPSS) for Windows version 11.5. P $<0.05$ was considered statistically significant.

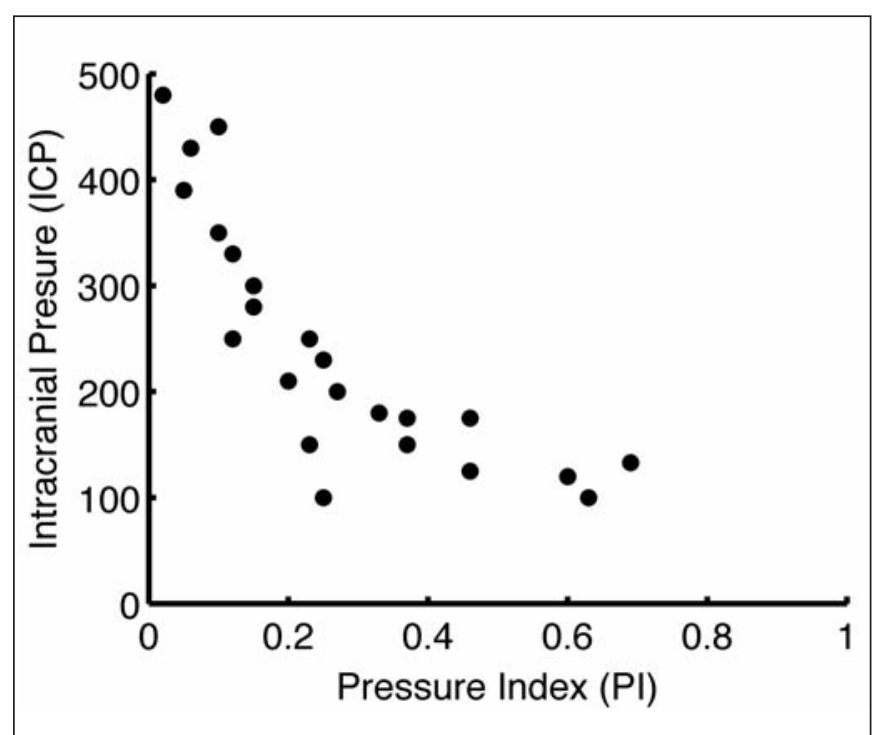

Figure 3: Correlation between PI and ICP in patients with focal lesions of $C N S(p<0.01 ; r=-0.912 ; n=23)$

\section{RESULTS}

The 62 patients included 34 males and 28 females. Mean age was $37.27 \pm 16.53$ years. Patients with 16 different diseases were admitted (see Table 1). The most common disease among our patients was tuberculous meningitis $(\mathrm{n}=12,19.4 \%)$. The mean ICP was $239.74 \pm 116.25 \mathrm{mmH}_{2} \mathrm{O}\left(70-500 \mathrm{mmH}_{2} \mathrm{O}\right)$, and $52.9 \%$ of patients had increased ICP. The mean PI was $0.29 \pm 0.20$ (0.02-0.85). The Spearman rank test showed that there was a significant negative correlation between PI and ICP $(r=-0.849$, $\mathrm{p}<0.01$, Figure 1). The data from the patients with diffuse lesions of CNS and focal lesions were analyzed separately. The results showed there were significant negative correlations between PI and ICP in both groups $(\mathrm{r}=-0.815,-0.912 ; \mathrm{p}<0.01$; Figure 2, 3)

Table 2: Follow-up PI and ICP data of two patients with benign intracranial hypertension (Patient 1) and Tuberculous meningitis (Patient 2)

\begin{tabular}{|c|c|c|c|c|}
\hline \multirow{2}{*}{ Follow up } & \multicolumn{2}{|c|}{ Patient1 ${ }^{*}$} & \multicolumn{2}{|l|}{ Patient $2^{\#}$} \\
\hline & $\mathrm{ICP}\left(\mathrm{mm} \mathrm{H}_{2} \mathrm{O}\right)$ & PI & $\mathrm{ICP}\left(\mathrm{mm} \mathrm{H}_{2} \mathrm{O}\right)$ & PI \\
\hline First & 460 & 0.04 & 400 & 0.06 \\
\hline Second & 400 & 0.08 & 339 & 0.18 \\
\hline Third & 225 & 0.26 & 260 & 0.28 \\
\hline Fourth & 175 & 0.44 & 200 & 0.27 \\
\hline Fifth & 150 & 0.53 & 150 & 0.41 \\
\hline
\end{tabular}

There were good correlations between PI and ICP in Patient $1(\mathrm{p} *<0.01 ; \mathrm{r}=-0.960 ; \mathrm{n}=5)$ and Patient $2(\mathrm{p} \#<0.01 ; \mathrm{r}=-0.997 ; \mathrm{n}=5)$. 
One patient with benign intracranial hypertension and another patient with tuberculous meningitis received long-term followup (Table 2). There were negative correlations between PI and ICP in both patients $(r=-0.960,-0.997 ; \mathrm{p}<0.01)$.

\section{Discussion}

In this study, we analyzed the EEG power spectrum in patients with CNS disorders and calculated the PI with selfdesigned software. Meanwhile, ICP was measured by LP. We found that PI was correlated with ICP. In addition, a good correlation between PI and ICP also existed in both groups of patients with diffuse lesions and focal lesions of the CNS.

The function and metabolism of the brain depend on a continuous supply of cerebral blood. In order to maintain normal metabolism and function of the brain tissue, CBF must be kept relatively stable. When ICP increases progressively, cerebrovascular autoregulatory dysfunction may occur. Cerebral blood flow will decrease with the reduction of perfusion pressure, which will result in a series of changes in brain tissues due to hypoxic-ischemic injury, including continuous depolarization of cell membrane, reduced ability to generate cellular excitation, increased intracellular free calcium, apoptosis, and reduced neurotransmitter synthesis. Insufficient adenosine triphosphate and acidosis can exacerbate the damage of brain cells. The arrangement of neurons becomes disordered and the synchronization of excitation is reduced after cerebral injury, so the slow-wave components of the EEG increase. When ICP increases to a similar level of blood pressure, cerebral circulation will cease along with disappearance of EEG activity and brain death. Since ICP change results in EEG change, it is rational that certain EEG-derived parameters may be able to reflect ICP change.

In this study, we recorded EEG and obtained the parameters, including median frequency and delta ratio, by EEG power spectrum analysis and, from this, PI was calculated. The results showed that PI was correlated with $\operatorname{ICP}(r=-0.849, \mathrm{p}<0.01)$. In addition, PI and ICP had good correlation in both groups of patients with the diffuse lesions and focal lesions of CNS, which suggested that despite the focal brain lesion itself might have impact on EEG activity, PI still correlated with ICP in patients suffering from a variety of different neurological diseases. Dynamic measurement of the same patient also showed that PI correlated well with ICP, which suggested that PI might be used instead of frequent LPs, at least for patients who require followup, such as patients with benign intracranial hypertension, and postoperative hydrocephalus. Moreover, based on the good correlation between PI and ICP, we speculate that continuous PI monitoring has potential to detect the real-time ICP dynamic, especially disproportionate increase in ICP which indicates decreased intracranial compliance or craniospinal compensatory ability $(\mathrm{CCA})^{17-19}$. Detecting CCA is helpful to guide medical management of individuals with acute brain injury because intervention during this time window may reduce potential risk for secondary insult ${ }^{20-22}$. Therefore, the ability to detect CCA by PI monitoring should be confirmed in a future study.

Our study has some limitations. (1) ICP measured by LP may not be consistent with ICP measured by a more direct intracranial probe in some situations, such as arachnoid adhesions or spinal canal stenosis leading to obstruction of cerebrospinal fluid circulation. In other words, ICP obtained by LP might not reflect the true ICP in these cases. (2) A certain kind of curve rather than a linear correlation may exist between the PI and ICP, therefore quantitative mathematical relationship between the PI and ICP remains to be further clarified. (3) The EEG information acquired might be insufficient because only a few electrodes used, which might influence the PI value. It may be useful during future studies to acquire more EEG information by adding additional electrode locations. (4) We conducted onetime EEG acquisition and simultaneous ICP measurement. The stability or repeatability of PI could not be confirmed. ICP monitoring by direct intracranial fiberoptic probe and continuous EEG acquisition may help to solve this problem. (5) Though ICP and PI had good correlation in both groups of patients with diffuse lesions and focal lesions of CNS, some other clinical factors such as sedatives, narcotics, or paralytic agents may affect the electrical activity of brain cells and PI values may not reflect ICP accurately in that case. This may be one of the defects of this method.

In summary, our preliminary study suggested that analysis of some specific parameters from EEG power spectrum might reflect the ICP, and is worthy of further study.

\section{ACKNOWLEDGEMENT}

The authors thank the Science and Technology Commission of Chongqing for their financial support to the present work (cstc2011jjA0084).

\section{REFERENCES}

1. Czosnyka M, Pickard JD. Monitoring and interpretation of intracranial pressure. J Neurol Neurosurg Psychiatry. 2004;75 (6):813-21.

2. Lundberg N. Continuous recording and control of ventricular fluid pressure in neurosurgical practice. Acta Psychiatr Neurol Scand. 1960;36(suppl 149):1-193.

3. Ghajar J. Intracranial pressure monitoring techniques. New Horiz. 1995;3(3):395-9.

4. Lozier AP, Sciacca RR, Romagnoli MF, et al. Ventriculostomyrelated infections: a critical review of the literature. Neurosurgery. 2008;62(Suppl 2):688-700.

5. Mayhall CG, Archer NH, Lamb VA, et al. Ventriculostomy-related infections. A prospective epidemiologic study. N Engl J Med. 1984;310(9):553-9.

6. Steiner LA, Andrews PJD. Monitoring the injured brain: ICP and CBF. Br J Anaesth. 2006;97(1):26-38.

7. Petkus V, Ragauskas A, Jurkonis R. Investigation of intracranial media ultrasonic monitoring model. Ultrasonics. 2002;40(1-8): 829-33.

8. Schmidt B, Klingelhofer J, Schwarze JJ, et al. Noninvasive prediction of intracranial pressure curves using transcranial Doppler ultrasonography and blood pressure curves. Stroke. 1997;28(12):2465-72.

9. Schoser BG, Riemenschneider N, Hansen HC. The impact of raised intracranial pressure on cerebral venous hemodynamics: a prospective venous transcranial Doppler ultrasonography study. J Neurosurg. 1999;91(5):744-9.

10. Reid A, Marchbanks RJ, Bateman DE, et al. Mean intracranial pressure monitoring by an audiological technique-a pilot study. J Neurol Neurosurg Psychiatry. 1989;52(5):610-2.

11. Shimbles S, Dodd C, Banister K, et al. Clinical comparison of tympanic membrane displacement with invasive ICP measurements. Physiol Meas. 2005;26(6):1085-92.

12. Friedman D, Claassen J, Hirsch LJ. Continuous electroencephalogram monitoring in the intensive care unit. Anesth Analg. 2009;109(2):506-23. 
13. Otto KA. EEG power spectrum analysis for monitoring depth of anaesthesia during experimental surgery. Lab Anim. 2008;42(1): 45-61.

14. Czigler B, Csikós D, Hidasi Z, et al. Quantitative EEG in early Alzheimer's disease patients-power spectrum and complexity features. Int J Psychophysiol. 2008;68(1):75-80.

15. Drummond JC. Monitoring depth of anesthesia: with emphasis on the application of the Bispectral Index and the middle latency auditory evoked response to the prevention of recall. Anesthesiology. 2000;93(3):876-82.

16. Balestreri $M$, Czosnyka $M$, Hutchinson $P$, et al. Impact of intracranial pressure and cerebral perfusion pressure on severe disability and mortality after head injury. Neurocrit Care. 2006;4 (1):8-13.

17. Contant CF Jr. Robertson CS, Crouch J, et al. Intracranial pressure waveform indices in transient and refractory intracranial hypertension. J Neurosci Methods. 1995;57(1): 15-25
18. Rauch ME, Mitchell PH, Tyler ML. Validation of risk factors for the nursing diagnosis decreased intracranial adaptive capacity. J Neurosci Nurs. 1990;22(3):173 -8

19. Fan JY, Kirkness C, Vicini P, et al. An approach to determining intracranial pressure variability capable of predicting decreased intracranial adaptive capacity in patient with traumatic brain injury. Biol Res Nurs. 2010;11(4):317-24.

20. Chesnut RM, Marshall LF, Klauber MR, et al. The role of secondary brain injury in determining outcome from severe head injury. J Trauma. 1993;34(2):216-22.

21. Lang EW, Chesnut RM. Intracranial pressure and cerebral perfusion pressure in severe head injury. New Horiz. 1995;3(3):400-9.

22. Resnick DK, Marion DW, Carlier P. Outcome analysis of patients with severe head injuries and prolonged intracranial hypertension. J Trauma. 1997;42(6):1108-11. 